\title{
University of Massachusetts Amherst
}

From the SelectedWorks of William M. Irvine

January 1, 1989

\section{Scattering of Light by Stochastically Rough Particles}

J.I. Peltoniemi

K. Lumme

K. Muinonen

William M. Irvine, University of Massachusetts - Amherst 


\title{
Scattering of light by stochastically rough particles
}

\author{
Jouni I. Peltoniemi, Kari Lumme, Karri Muinonen, and William M. Irvine
}

\begin{abstract}
The single particle phase function and the linear polarization for large stochastically deformed spheres have been calculated by Monte Carlo simulation using the geometrical optics approximation. The radius vector of a particle is assumed to obey a bivariate lognormal distribution with three free parameters: mean radius, its standard deviation and the coherence length of the autocorrelation function. All reflections/refractions which include sufficient energy have been included. Real and imaginary parts of the refractive index can be varied without any restrictions. Results and comparisons with some earlier less general theories are presented. Applications of this theory to the photometric properties of atmosphereless bodies and interplanetary dust are discussed.
\end{abstract}

\section{Introduction}

In nature most particles are irregular. These particles scatter light in a way different from regular ones. The scattering from irregular particles is, however, poorly characterized.

The exact starting point for calculating all electrodynamic problems is Maxwell's equations. In the case of scattering by particles, we have to solve a boundary value problem. This can be done easily for some regular forms only. 1,2

For random surfaces that deviate only slightly from a regular form, some perturbation methods can be used. Schiffer ${ }^{3}$ has calculated the reflection from a perturbed sphere and Bass and Fuks ${ }^{4}$ presented some methods to calculate scattering from random surfaces.

To calculate the scattering from macroscopically deformed particles one must apply more approximate methods. For large particles geometrical optics can be used. For example, Schiffer and Thielheim ${ }^{5}$ used geometrical optics to find analytical forms for single reflection, multiple scattering from a surface and for light passing through the particle using shadowing theory. They failed, however, to treat the multiple scattering correctly.

Another approach is that by Mukai et al. 6 They assumed a large rough sphere and used geometrical optics and 1-D radiative transfer theory to calculate

William Irvine is with University of Massachusetts, Five College Radio Astronomy Observatory, Amherst, Massachusetts 01003; the other authors are with University of Helsinki, Observatory \& Astrophysics Laboratory, Tähtitornimäki, 00130 Helsinki, Finland.

Received 16 September 1988.

0003-6935/89/194088-08\$02.00/0.

(C) 1989 Optical Society of America. the scattering by a rough surface and integrated over the sphere. They assumed that the absorption is so high that no light travels through the particle. These simplifying assumptions restrict the generality of their model to the point where we believe it is not very accurate for typical rough particles. Some additional methods are listed in Schuerman ${ }^{7}$ and Giese and Lamy. ${ }^{8}$

In this paper we assume the geometrical optics regime. Using a Monte Carlo simulation we have been able to develop a model with which we can investigate both dielectric and absorbing particles with no limit on the refractive index and with a well controlled roughness scale.

The underlying optical theory is presented first. Then we define the particle model and give those formulas with which this specific problem is solved.

In Sec. IV we analyze the results and fit the phase functions to an analytic function. In Sec. V the results are compared with some experimental results and with earlier theories. Lastly we discuss astronomic applications, estimate the reliability of the present model, and give some suggestions for further development.

\section{Ray Tracing Model}

In what follows we assume that the particle is large enough to allow the application of geometrical optics. This approximation is valid if the size parameter $x=$ $k D \gg 1$, where $k=2 \pi / \lambda$ is the wavenumber, $\lambda$ is the wavelength, and $D$ is a (smallest) characteristic dimension of the particle. Polarization is taken into account by using Stokes parameters $I=(I, Q, U, V) .1,9$

We express the scattering in the form

$$
\mathbf{I}_{s}(\Omega)=\frac{\sigma_{\text {ext }} \tilde{\omega}_{0}}{r^{2}} \int d^{2} \Omega^{\prime} \frac{\mathbf{P}(\theta)}{4 \pi} \mathbf{I}_{i}\left(\Omega^{\prime}\right),
$$

where $\mathbf{I}_{i}$ is the Stokes vector of the incoming radiance, $\mathbf{I}_{s}$ is the scattered one, $\sigma_{\text {ext }}$ is the extinction cross sec- 
tion, $\tilde{\omega}_{0}$ is single scattering albedo, $r$ is the distance from the scatterer, $\theta$ is the scattering angle $(\cos \theta=\Omega$. $\left.\Omega^{\prime}\right)$, and $\mathbf{P}$ is the $4 \times 4$ phase matrix, ${ }^{9}$ whose first element is normalized by

$$
\int_{4 \pi} d^{2} \Omega \frac{P_{11}(\theta)}{4 \pi}=1
$$

The equation is valid in the scattering region, i.e., when $r \gg a$, where $a$ is the largest dimension of the scatterer.

The path of the ray is assumed to be defined by a Markov process (that means that the probability that the ray can advance from point $\mathbf{r}$ to $\mathbf{r}+d \mathbf{r}$ depends only on the situation at $\mathbf{r}$, not on the previous history) so that we can write the whole scattering in the form of an integro-differential equation. The procedure is in essence analogous to the classical radiative transfer theory, but now the scatterings are reflections and refractions from the boundary which separates regions of different refractive indices $m$. Also the propagation probability for a photon is now nonisotropic. The path of the ray is characterized by a position vector $\mathbf{r}$ and a ray direction vector $\Omega(|\Omega|=1)$. The probability that the ray will be either scattered or absorbed in distance $d l$ is $\beta d l$, where $\beta$ is the extinction coefficient. This can be divided into scattering and absorption coefficients as $\beta=\beta_{s}+\beta_{a}$. The matrix $\mathrm{W}(\Omega, m)$ describes the probability that the ray will be scattered into the direction $\Omega$ and medium $m$ and does the necessary transformations for polarization. The emitted radiation is described by the source distribution $\Psi$, which for the current case is a plane wave source $\Psi(\mathbf{r}, \Omega)=\pi F_{0} \delta\left(z-z_{0}\right) \delta\left(\Omega-\Omega_{0}\right)$. With this notation we get the equation ${ }^{9,10}$

$\beta(\mathbf{r}, \mathbf{\Omega}, m) \mathbf{I}(\mathbf{r}, \mathbf{\Omega}, m)+\mathbf{\Omega} \cdot \nabla \mathbf{I}(\mathbf{r}, \mathbf{\Omega}, m)$

$$
=\sum_{m^{\prime}} \int d \mathbf{\Omega}^{\prime} \mathbf{W}\left(\mathbf{\Omega}, m \mid \mathbf{r}, \boldsymbol{\Omega}^{\prime}, m^{\prime}\right) \beta_{s}\left(\mathbf{r}, \Omega^{\prime}, m^{\prime}\right) \mathbf{I}\left(\mathbf{r}, \boldsymbol{\Omega}^{\prime}, m^{\prime}\right)+\Psi(\mathbf{r}, \Omega, m),
$$

where the integration goes over the angle and the sum is over the refractive index values $m^{\prime}$ of all the different media involved. This is just the Boltzmann equation for radiative transfer. This can be converted to an integral equation

$$
\mathbf{I}=\hat{\mathbf{R I}}+\mathbf{I}_{0},
$$

where $\hat{\mathbf{K}}$ is the integral operator

$$
\begin{aligned}
\widehat{\mathbf{K}}= & \sum_{m^{\prime}} \int d \mathbf{\Omega}^{\prime} d \mathbf{r}^{\prime} \exp \left\{-\left[\tau(\mathbf{r}, \mathbf{\Omega}, m)-\tau\left(\mathbf{r}^{\prime}, \Omega, m\right)\right]\right\} \beta\left(\mathbf{r}^{\prime}, \Omega^{\prime}, m^{\prime}\right) \\
& \times \mathbf{W}\left(\mathbf{\Omega}, m \mid \mathbf{r}^{\prime}, \mathbf{\Omega}^{\prime}, m^{\prime}\right) \delta\left(\mathbf{r}^{\prime}-\mathbf{r}+\left|\mathbf{r}-\mathbf{r}^{\prime}\right| \mathbf{\Omega}\right), \\
I_{0}= & \int d \mathbf{r}^{\prime} \exp \left\{-\left[\tau(\mathbf{r}, \mathbf{\Omega}, m)-\tau\left(\mathbf{r}^{\prime}, \mathbf{\Omega}, m\right)\right]\right\} \\
& \times \delta\left(\mathbf{r}^{\prime}-\mathbf{r}+\left|\mathbf{r}^{\prime}-\mathbf{r}\right| \mathbf{\Omega}\right) \Psi\left(\mathbf{r}^{\prime}, \Omega, m\right),
\end{aligned}
$$

where $\tau$ is the optical depth $\tau(\mathbf{r}, \Omega, m)=\int_{-\infty}^{0} d l(\beta(\mathbf{r}+$ $\Omega l, \Omega, m)$. Equation (4) has the formal order of scattering solution

$$
\begin{aligned}
\mathbf{I}(\mathbf{r}, \Omega, m) & =\sum_{n} \hat{\mathbf{K}}^{n} \mathbf{I}_{0} \\
& =\mathbf{I}_{0}+\mathbf{I}_{1}+\mathbf{I}_{2}+\mathbf{I}_{3}+\mathbf{I}_{4}+\ldots \\
& =\mathbf{I}_{0}+\mathbf{I}_{s} .
\end{aligned}
$$

The phase matrix can now in principle be obtained from Eqs. (1)-(7). However, the formal solution contains multidimensional integrals that are impossible to calculate analytically. We found it most convenient to solve the problem with Monte Carlo simulation for ray tracing. ${ }^{10,11}$

The first step in this is the estimation of the free path length $L$ that the ray travels between scatterings. This is solved numerically from the equation $\tau(\mathbf{r}+\Omega L)$ $=\tau(\mathbf{r})+\ln (\boldsymbol{\Xi})$, where $\Xi$ is a random number distributed uniformly between 0 and 1 .

The second step is the simulation of the scattering process, which contains the selection of the orientation of a surface element at the interaction point, the selection between reflection and refraction, the calculation of the new direction, and the change of the Stokes vector. The distribution of the surface element depends on the particle model which is described in the next section. The selection between reflection and refraction is made by weighting with reflectivity and transmissivity (Fresnel coefficients), and the optical processing is simply a multiplication with the reflection or the transmission matrix and the appropriate rotation matrices.

We have not usually included diffraction in these results. Thus the forward scattering is not accurate for $\theta \approx 5^{\circ}$. For many applications this error is negligible, because for large particles the diffraction spike is very narrow and can be considered to be part of the unscattered radiation. Only in one case, where we compare our results to experiment (Fig. 6), have we taken diffraction into account. Even there the treatment is rather approximate, because we have assumed the projection of the particle to be circular and then used the standard circular aperture scalar diffraction theory ${ }^{12,1}$ averaged over a size distribution.

\section{Particle Model}

Our starting point is a homogeneous sphere which we deform stochastically. Assume that the probability density for the distance $h$ from the center of the particle to the surface follows the lognormal distribution

$$
f(h) d h=\frac{d h}{\sqrt{2 \pi} \beta h} \exp \left[-\left[\ln (h / a)+1 / 2 \beta^{2}\right]^{2} / 2 \beta^{2}\right],
$$

where $a$ is the mean radius, $\beta^{2}=\ln \left(1+\sigma^{2}\right)$, and $\sigma$ is the normalized standard deviation.

To specify the particle shape completely, we also need to know the autocorrelation function of the surface between two adjacent points. To keep the model simple it is assumed that the correlation depends only on the angular distance between these two points. At short distances we can use a second order Taylor expansion

$$
C(\epsilon)=1-\frac{1}{2} \frac{\epsilon^{2}}{\xi^{2}}-\ldots,
$$

where $\epsilon$ is the angular distance between the points and $\xi$ is the correlation angle (in radians). From these definitions we can determine the probability density 
function for the slope $(\gamma)$ of a surface element with respect to the radius ${ }^{4}$

$$
g_{1}\left(\tan \gamma_{1}\right) d \tan \gamma_{1}=\frac{d \tan \gamma_{1}}{\sqrt{2 \pi} \rho} \exp \left(-\tan ^{2} \gamma_{1} / 2 \rho^{2}\right),
$$

where $\rho=2 \sigma / \xi \sqrt{1+\sigma^{2}}$ is the standard deviation of the slopes. Equation (10) gives the slope only in one dimension. Because we assumed no azimuthal dependence on the correlation function, the 2-D distribution is simply the product of two 1-D distributions $\left(\tan ^{2} \gamma=\right.$ $\left.\tan ^{2} \gamma_{1}+\tan ^{2} \gamma_{2}\right)$. We obtain

$$
g(\tan \gamma, \zeta) d \tan \gamma d \zeta=\frac{\tan \gamma d \tan \gamma d \zeta}{2 \pi \rho^{2}} \exp \left(-\tan ^{2} \gamma / 2 \rho^{2}\right),
$$

where $\zeta$ is the azimuth. In Fig. 1 we present some computer-generated rough particles.

Physical and numerical reasons limit the sensible values of $\sigma$ to $0.002-0.5$ and of $\rho$ to 0.002-2.0. An increase of $\rho$ above this does not give any natural kind of roughness, because the slope is always limited between 0 and $90^{\circ}$ and with very high $\rho$ we get a surface where the mean slope is near $90^{\circ}$. The assumption that the path of a ray is Markovian prohibits all long range correlations. In other words the mean free path length must be longer than the correlation length and thus $\sigma<\rho$.

There are no essential restrictions on the refractive index, as long as it is a scalar. One difficulty arises when the imaginary part of the refractive index is high, because then the internal wave is inhomogeneous. We did not find it necessary to treat this properly ${ }^{19,4}$ because, when the inhomogeneity is large enough to produce visible effects $[\operatorname{Im}(m) \gg 0.01]$, the absorption is so high that under the assumption of geometrical optics $(x \gg 1)$ in practice all the energy entering the particle is absorbed.

Next we derive an expression for the scattering coefficient $\beta_{s}$. This is the probability that the ray, which at point $\mathbf{r}$ is outside (inside) the particle, will meet the surface in the distance $d l$ or that the radius of the surface at point $\mathbf{r}+\Omega d l$ is greater (smaller) than $\mid \mathbf{r}+$ $\Omega d l \mid{ }^{13} \quad$ For this to happen the normal of the surface element must be tilted toward the coming ray, or $\cot \psi$ $<\tan \gamma_{1}<\infty$ and the radius of the surface must be in $[r$ $-d h, r]$, where $d h=d l\left(-\cos \psi+\sin \psi \tan \gamma_{1}\right)$. This gives for a ray approaching the surface from outside

$$
\beta_{s}\left(\mathbf{r}, \Omega, m_{1}\right) d l=\frac{\int_{\cot \psi}^{\infty} d \tan \gamma_{1} d h f(r) g_{1}\left(\tan \gamma_{1}\right)}{\int_{0}^{r} d h f(h)},
$$

which reduces to

$$
\beta_{s}\left(\mathbf{r}, \Omega, m_{1}\right) d l=\frac{f(r)}{F(r)}\left[\rho^{2} g_{1}(\cot \psi)+\cot \psi G_{1}(\cot \psi)\right] \sin \psi d l,
$$

and for a ray inside the particle

$$
\beta_{s}\left(\mathbf{r}, \Omega, m_{2}\right) d l=\frac{f(r)}{1-F(r)}\left[\rho^{2} g_{1}(\cot \psi)-\cot \psi G_{1}(-\cot \psi)\right] \sin \psi d l,
$$

where $\cos \psi=\mathbf{r} \cdot \Omega / r ; f$ and $g$ are the probability densities for radius and slope of the surface [Eqs. (8) and

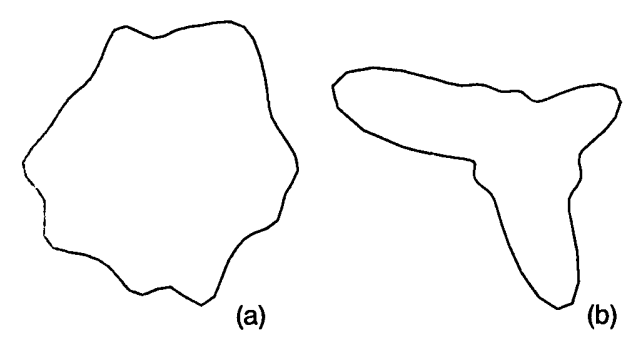

Fig. 1. Computer-generated silhouettes of typical rough particles: (a) $\sigma=0.1, \rho=0.5$; (b) $\sigma=0.5, \rho=1.5$, where $\sigma$ is the normalized standard deviation of the radius and $\rho$ is the standard deviation of the slope.

(10)] and $F, G$ are the respective distribution functions $F(r)=\int_{-\infty}^{r} d h f(h), G(x)=\int_{-\infty}^{x} d x g(x)$. From these the optical density can be calculated numerically or analytically when the curvature effects of the spherical geometry are negligible. The distribution for the freepath length can then be computed.

The effective distribution $g_{\text {eff }}$ for the slope, with the condition that the ray comes from direction $\psi$, is

$$
g_{\text {eff }}(\tan \gamma \mid \psi) d \tan \gamma d \zeta=\frac{d \tan \gamma d \zeta}{Q(\cot \psi)} \frac{\cos \iota}{\cos \gamma} g(\tan \gamma),
$$

where $Q$ is

$$
Q(t)=\int_{-\infty}^{t} d \tan \gamma d \zeta \frac{\cos \iota}{\cos \gamma} g(\tan \gamma) .
$$

The factor $\cos \iota=-\cos \psi \cos \gamma+\sin \psi \sin \gamma \cos \zeta$ is the fraction of the surface element seen by the ray, and $1 /$ $\cos \gamma$ gives the ratio between the real surface element and its projection to the horizontal level.

The matrix W in Eqs. (3) and (5) is now

$$
\begin{aligned}
\mathbf{W}\left(\boldsymbol{\Omega}, m \mid \mathbf{r}, \boldsymbol{\Omega}^{\prime}, m^{\prime}\right)= & \mathbf{K}\left(\Phi_{2}\right)\left\{\begin{array}{l}
\mathbf{R} \\
\mathbf{T}
\end{array}\right\}\left(\alpha_{i}, \alpha_{t}, m, m^{\prime}\right) \mathbf{K}\left(\Phi_{1}\right) \\
& \times g_{\text {eff }}(\tan \eta \mid \psi) \frac{d \tan \gamma d \zeta}{d \mathbf{\Omega}^{\prime}},
\end{aligned}
$$

where $\Omega$ is the direction of the scattered ray and $\Omega^{\prime}$ is the direction of the incident ray. The $\mathbf{K}$ are rotation matrices that are needed first to rotate the reference plane of the Stokes vector to the current scattering plane that is defined by the normal of the surface element and the direction of ray $\left(\mathbf{n}, \Omega^{\prime}\right)$ and then to rotate back to the standard plane that is defined by the original and present ray $\left(\Omega_{0}, \Omega\right),{ }^{9,10}$

$$
\mathbf{K}=\left[\begin{array}{cccc}
1 & 0 & 0 & 0 \\
0 & \cos 2 \Phi & \sin 2 \Phi & 0 \\
0 & -\sin 2 \Phi & \cos 2 \Phi & 0 \\
0 & 0 & 0 & 1
\end{array}\right]
$$

The $\Phi$ are rotation angles

$$
\begin{aligned}
& \cos \Phi_{1}=\frac{\mathbf{n} \cdot \Omega_{0}+\Omega^{\prime} \cdot \Omega_{0} \cos \alpha_{i}}{\sqrt{1-\Omega^{\prime} \cdot \Omega_{0}} \sin \alpha_{i}}, \\
& \sin \Phi_{1}=\operatorname{sign}\left(\Omega_{0} \cdot \mathbf{n} \times \Omega^{\prime}\right) \sqrt{1-\cos ^{2} \Phi_{1}},
\end{aligned}
$$

and

$$
\cos \Phi_{2}=\frac{\mathbf{n} \cdot \boldsymbol{\Omega}_{0}+\mathbf{\Omega} \cdot \boldsymbol{\Omega}_{0} \cos \alpha_{i}}{\sqrt{1-\boldsymbol{\Omega} \cdot \mathbf{\Omega}_{0}} \sin \alpha_{i}},
$$




$$
\sin \Phi_{1}=-\operatorname{sign}\left(\Omega_{0} \cdot \mathbf{n} \times \Omega\right) \sqrt{1-\cos ^{2} \Phi_{2}},
$$

In the case of reflection $\left(m=m^{\prime}\right)$ the incident angle is

$$
\cos \alpha_{i}=\sqrt{\frac{1-\Omega \cdot \Omega^{\prime}}{2}},
$$

and the normal

$$
\mathbf{n}=\frac{\mathbf{\Omega}-\boldsymbol{\Omega}^{\prime}}{2 \cos \alpha_{i}} .
$$

In refraction $\left(m \neq m^{\prime}\right)$ we have for incident and refraction angles

$$
\begin{aligned}
\cos \alpha_{i} & =\frac{\left|m \boldsymbol{\Omega} \cdot \boldsymbol{\Omega}-m^{\prime}\right|}{\sqrt{m^{2}+m^{\prime 2}-2 m m^{\prime} \Omega \cdot \Omega^{\prime}}}, \\
\cos \alpha_{t} & =\frac{\left|m^{\prime} \Omega \cdot \Omega^{\prime}-m\right|}{\sqrt{m^{2}+m^{\prime 2}-2 m m^{\prime} \Omega \cdot \Omega^{\prime}}}, \\
\mathbf{n} & =\frac{m^{\prime} \Omega^{\prime}-m \Omega}{m \cos \alpha_{i}-m^{\prime} \cos \alpha_{t}} .
\end{aligned}
$$

The reflection $\mathbf{R}$ and transmission $\mathbf{T}$ matrices with Fresnel coefficients $(r, t)^{19,1}$

$\mathbf{R}=\frac{1}{2}\left[\begin{array}{cccc}\left|r_{\|}\right|^{2}+\left|r_{\perp}\right|^{2} & \left|r_{\|}\right|^{2}-\left|r_{\perp}\right|^{2} & 0 & 0 \\ \left|r_{\|}\right|^{2}-\left|r_{\perp}\right|^{2} & \left|r_{\|}\right|^{2}+\left|r_{\perp}\right|^{2} & 0 & 0 \\ 0 & 0 & 2 \operatorname{Re}\left[r_{\|}^{*} r_{\perp}\right] & 2 \operatorname{Im}\left[r_{\|}^{*} r_{\perp}\right] \\ 0 & 0 & -2 \operatorname{Im}\left[r_{\|}^{*} r_{\perp}\right] & 2 \operatorname{Re}\left[r_{\|}^{*} r_{\perp}\right]\end{array}\right]$,

$\mathbf{R}=\frac{m \cos \alpha_{t}}{2 m^{\prime} \cos \alpha_{i}}\left[\begin{array}{cccc}\left|t_{\|}\right|^{2}+\left|t_{\perp}\right|^{2} & \left|t_{\|}\right|^{2}-\left|t_{\perp}\right|^{2} & 0 & 0 \\ \left|t_{\|}\right|^{2}-\left|t_{\perp}\right|^{2} & \left|t_{\|}\right|^{2}+\left|t_{\perp}\right|^{2} & 0 & 0 \\ 0 & 0 & 2 \operatorname{Re}\left[t_{\|}^{*} t_{\perp}\right] & 2 \operatorname{Im}\left[t_{\|}^{*} t_{\perp}\right] \\ 0 & 0 & -2 \operatorname{Im}\left[t_{\|}^{*} t_{\perp}\right] & 2 \operatorname{Re}\left[t_{\|}^{*} t_{\perp}\right]\end{array}\right]$.

The last factor in Eq. (17) is the Jacobian for changing the variables of $q$ from $\gamma, \zeta$ to $\Omega^{\prime}$. The connection between them is

$$
\begin{aligned}
& \cos \gamma=\mathbf{n} \cdot \mathbf{r} / r \\
& \cos \zeta=-\frac{\cos \psi \cos \gamma+\cos \alpha_{1}}{\sin \psi \sin \gamma} .
\end{aligned}
$$

\section{Summary of Results}

We have computed over seventy different cases by varying the parameters, in most cases with refractive indices $m=1.33$ or $m=1.55+i n^{\prime}$, where $n^{\prime}$ varies from 0 to 10 , which are thought to represent many typical materials.

We calculated the phase function $P_{11}$ and linear polarization $-P_{21} / P_{11}$. All other matrix elements could be calculated as well, but they are not so important in the most usual cases. We always used a few million input rays, and followed the ray until it came out or was absorbed (cutoff value for intensity $10^{-4}$ $10^{-5} I_{0}$ ). The accuracy of the simulation is proportional to the square root of the number of rays. The program gives an estimate for the error produced by the finite number of rays, and it is usually $<1 \%$ except in the backward direction, where it can be as large as $20-40 \%$; this error decreases rapidly to a few percent 2 or $3^{\circ}$ away from the exact backward direction. The use of a Markov process can give a little inaccuracy in the backward direction, because this method does not properly take into account the fact that the ray reflected directly back must of course advance through exactly the same way it came. Computing time with a VAX -8800 computer was $4-5 \mathrm{~h}$ for one case. We can summarize our results as:

Effect of small roughness With zero roughness we naturally have a sphere. When disturbing that very little $(\sigma, \rho<0.01)$ the first visible effects are that the rainbows move closer to each other and spread; see Fig. 2. With water droplets $(m=1.33)$ we get a small spike, or glory, in the backward direction. With increasing roughness the rainbows and spikes disappear. With spheres the polarization has a rapidly oscillating fine structure near the rainbows. This of course vanishes with roughness and the polarizing gets its typical shape with two maxima $\left(60-70^{\circ}\right.$ and $\left.120-160^{\circ}\right)$ and two minima $\left(40-50^{\circ}\right.$ and $\left.90-150^{\circ}\right)$. The increase of roughness moves all of them forward. All this is produced by roughness that corresponds to a particle which is very hard to distinguish from a sphere from the profile of the particle. An interesting observation is that the effect of perturbing the sphere very little is rather similar to averaged Mie results when decreasing the size parameter..$^{9}$ The explanation for this is that with smaller spheres the localization principle of geometrical optics does not hold and the rays are spread wider than the specific direction given by Snell's law. Of course the similarity disappears with larger roughness.

Effect of large roughness Really rough particles are illustrated in Fig. 3. The phase function varies smoothly in the middle and backward directions. The forward scattering is always strong, but large roughness weakens that. Rainbows, spikes, and other fine structures do not exist. With dielectric rough particles the polarization is small $(<10 \%)$ and no negative polarization in the backward direction is evident.

$\sigma$ vs $\rho$ The typical features of roughness can be achieved with many combinations of $\sigma$ and $\rho$. If we nonetheless try to separate their effects, we can say that $\rho$ is more responsible for decreasing polarization. The effect of $\sigma$ is most visible in the middle angles where it controls the absolute magnitude of the phase function.

Effect of the refractive index The effects of roughness become evident more rapidly for large refractive indices than for small ones, i.e., particles are optically rougher (Fig. 4). Decreasing the refractive index produces more forward scattering. With small refrctive index $(m \approx 1.6)$ the backward polarization maximum 

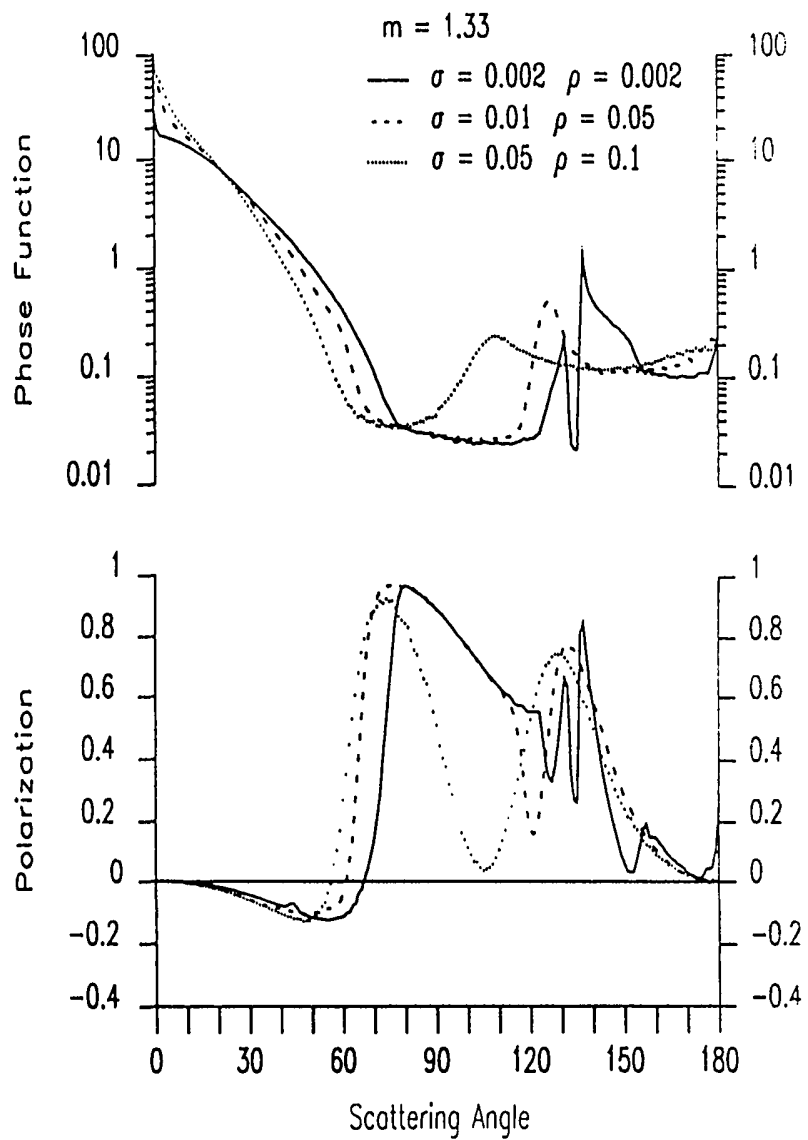

Fig. 2. Small deformations from the sphere. This figure illustrates clearly how fast even a small roughness changes the scattering behavior. The first particle is almost spherical; the only effects of roughness are a small opposition spike and the moving of rainbows closer to each other. The seocnd particle is slightly deformed and the third has visible irregularities. Note the great change in both the phase function and polarization.

tends to dominate, and with large $m(\widetilde{>} 1.6)$ the forward peak is more dominant.

Effect of absorption The increase of the absorption coefficient decreases the internal intensity and thus decreases the value of the phase function in the forward direction, as shown in Fig. 5. The polarization becomes stronger because the external reflections begin to dominate. As long as the imaginary part of the refractive index is small $(\ll 0.01)$, the results can be scaled keeping the product $\operatorname{Im}(m) x$ constant, but with a large imaginary part the Fresnel coefficients are changed and the reflectivity of the surface starts to increase. The large roughness decreases the albedo $\left(\tilde{\omega}_{0}\right)$. With very rough and highly absorbing particles there can occasionally be a small amount of negative polarization in the backward direction, caused by multiple external reflections, but this is not enough to explain any observed phenomena, like the negative polarization of atmosphereless bodies. With absorbing particles the difference between true spheres and rough particles is not as clear as with dielectric particles. Polarizations differ only when there is considerable multiple scattering. Because of shadowing the
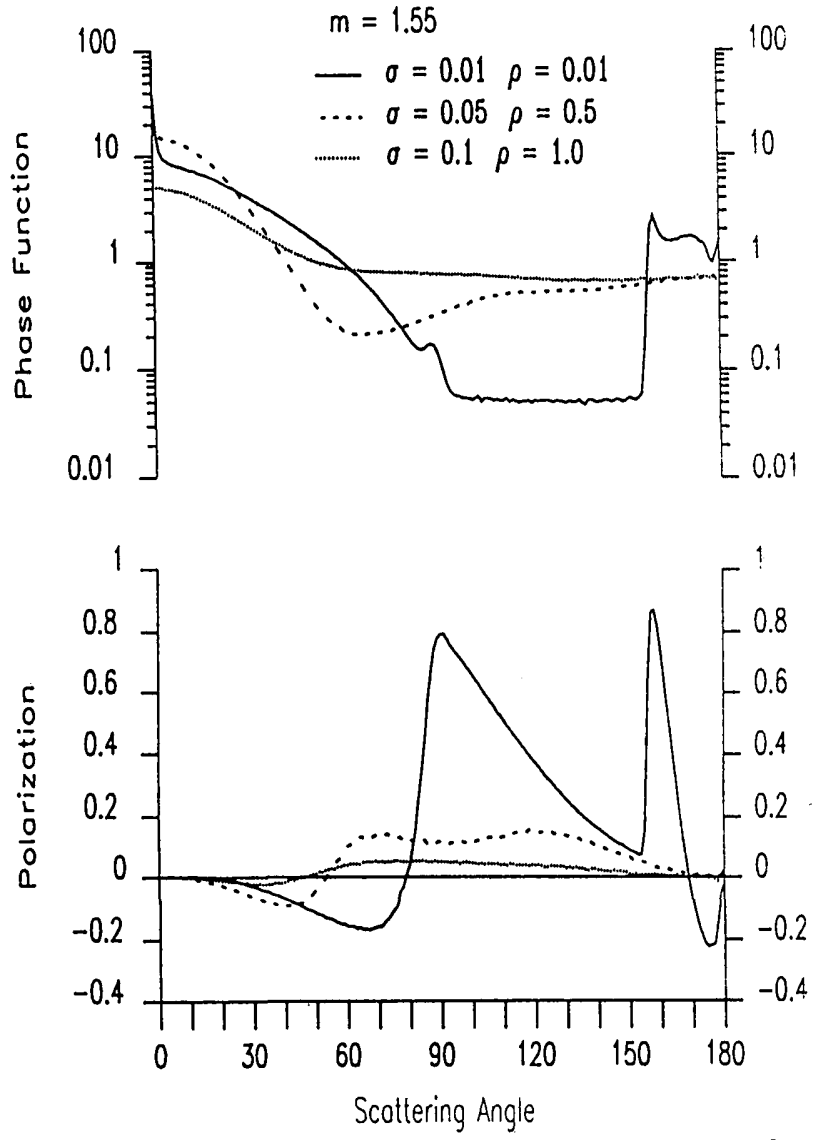

Fig. 3. Typical rough particles with $m=1.55$. As in Fig. 2 we have the nearly spherical particle first as a comparison base. The two other particles are intermediately and extremely rough. The difference between spherical and irregular particles is very clear. An additional increase of roughness does not give anything new within this theory.

phase function is a bit more backward oriented with rough particles than with smooth ones.

In many applications it is useful to have an analytical form of the phase function. The numerical calculations do not directly support any analytical forms, so we have to proceed in a different way. We have carefully selected among simple analytical functions with few free parameters, and then made a least-squares fit to numerical data to fix the parameters. The selected properly normalized function is

$$
P_{11}=\sum_{k=k_{\min }}^{k_{\max }} a_{k} \exp (k \cos \theta),
$$

where $a_{0}=1-\sum_{k \neq 0} a_{k} \sinh k / k$. We included terms from $k=-1$ to +4 , i.e., five free parameters. The advantages of this function are that it is easy to manipulate analytically, it is normalized, it is linear with the parameters so it can be fitted easily to data, and it describes the scattering behavior very well with all the refractive index values. The calculated parameters with error estimates are presented in Table I and in Figs. 6 and 7. At best the root mean square error is only a few percent. When the roughness is small, the phase functions become more complicated and the five 

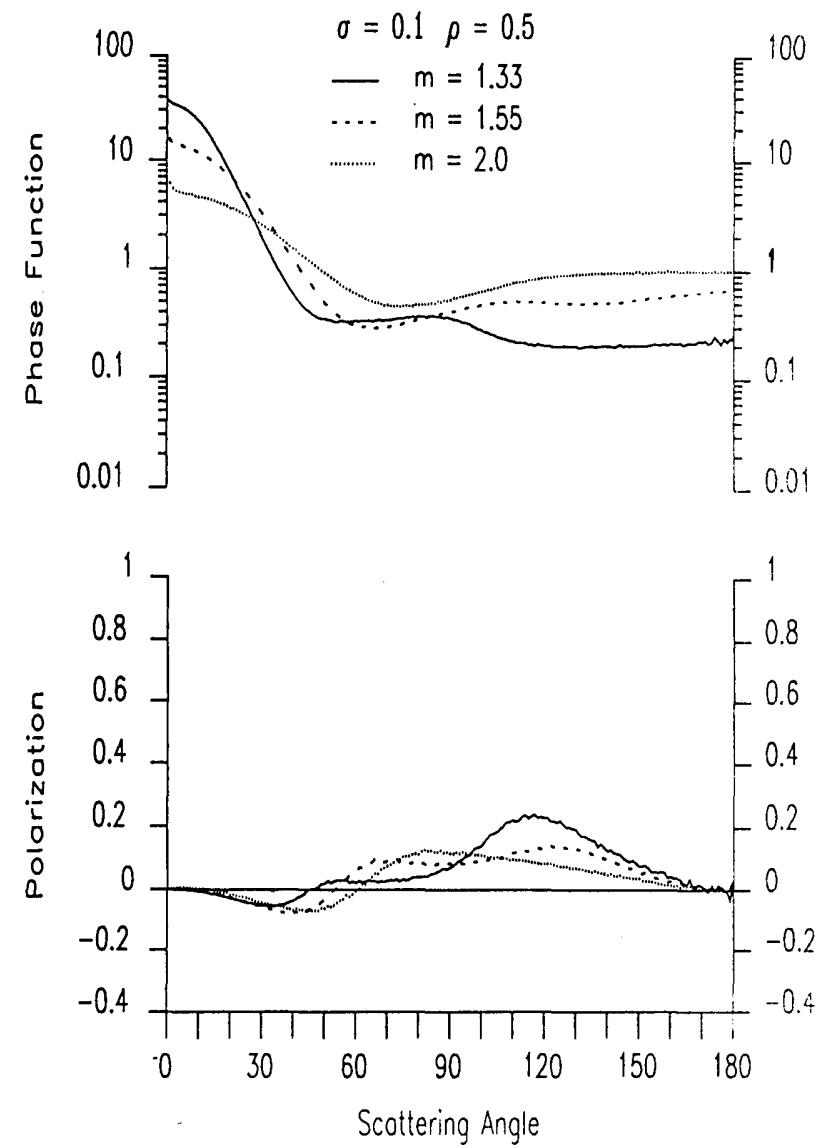

Fig. 4. Roughness of the particle is now fixed and the scattering has been calculated with different refractive indices.

parameter model can no longer provide good agreement; therefore more terms or a more complicated function are needed. For the sake of comparison, the rms error using a Mie scattering solution to approximate the solution of an irregular particle can be several hundred percent, and even the sum of two HenyeyGreenstein functions gives typically much more than $10 \%$ errors. With any polynomials about twice the number of terms (10) are needed to get the same accuracy as here.

We emphasize that these forms are only least square fits to numerical results and do not have any deeper theoretical character. Our purpose when presenting this function is solely to give a better approximation for those who still use isotropic, Henyey-Greenstein, or Mie phase functions to describe the unknown scatterers.

\section{v. Comparison with Experiments and Other Theoretical Models}

The calculated results may be compared with some experimental results. We have first used the results of Giese et $a l .{ }^{14}$ who measured fluffy rough particles with their microwave equipment (Fig. 6). Even though the size parameters are rather small $(x \sim 30)$, which makes the use of geometrical optics very doubtful, we still hope the comparison is useful. The roughness parameters were crudely estimated from the photographs
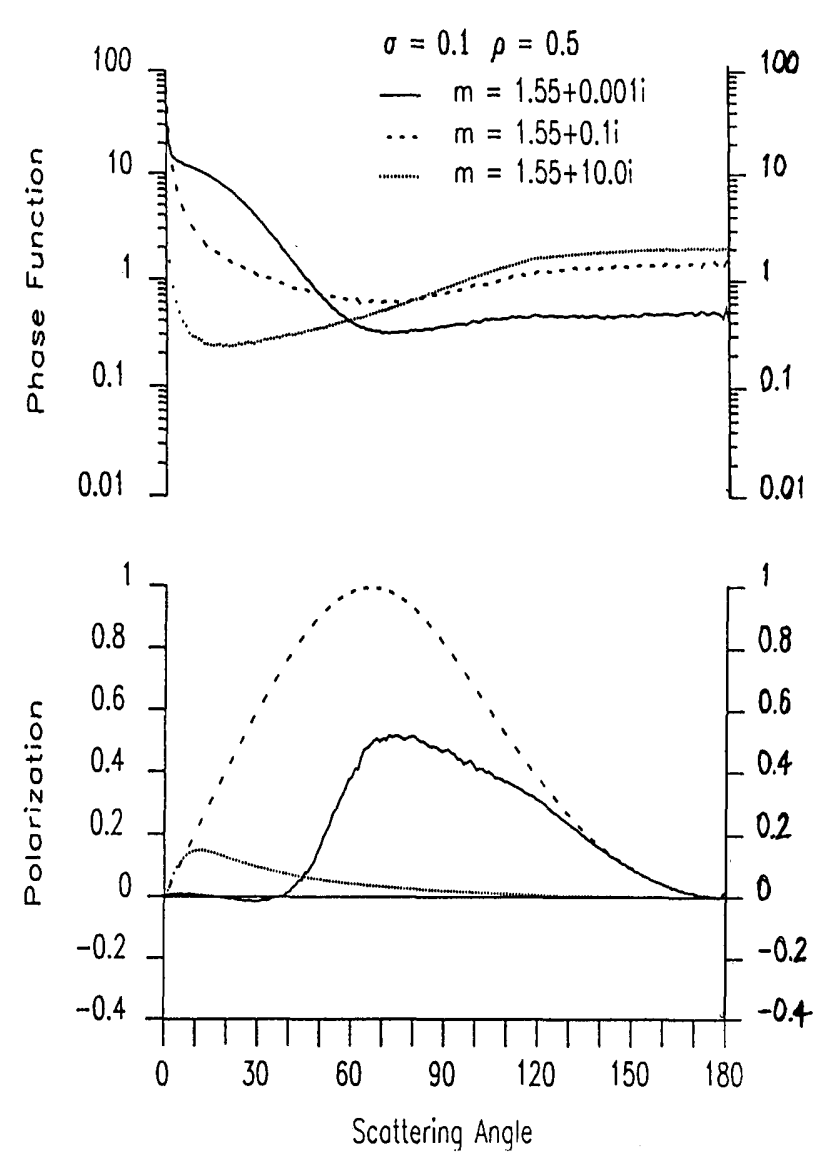

Fig. 5. Dependence of the phase function and the polarization on the imaginary part of the refractive index. The size parameter $x=$ 500 .

published in the same article. Taking diffraction into account we find that the agreement of overall behavior is good, but differences in detail remain.

Another experiment is that by Weiss-Wrana, ${ }^{15}$ in which she measured small particles with a laser. Here too we have to be careful in applying geometrical optics, because even though the particles are large, they have a fine structure just in the resonance region. The comparison for a dielectric quartz particle $(m=1.54, x$ $=150$ ) is shown in Fig. 7. The agreement for the phase functions is relatively satisfactory and for polarization it is amazingly good. That some small discrepancies still exist is most likely because we failed to reconstruct the properties of the particles completely. Another reason can be some wave optics effects caused by the microstructure of the particles.

If we compare our results with the calculations by Mukai et al. ${ }^{6}$ we note that, excluding some differences caused by diffraction, the results agree rather well. Our polarization is typically between Mukai's rough and smooth, which is in accordance with the selection of surface slope distributions.

For the verification of our model one should do more controlled measurements where the particle would be clearly in the geometrical optics region and where the roughness of the surface could be determined. 


\section{Discussion}

Geometrical optics is no doubt a rather reliable approximation when the particle is large, but at this point we cannot accurately define the lower limit for the size of the particle for geometrical optics to still hold. For spheres the limit for the size parameter is many hundreds, but the sphere is perhaps the most pathological case, and one can intuitively believe that stochastic particles obey geometrical optics much better. After all, geometrical optics is a random phase approximation, and thus random surfaces will make the phases even more random. The comparisons with experimental results support the last argument, but this may be as well good luck as good theory. One must keep in mind that in principle all the structures of the particle must be large, i.e., the surface elements from which the ray reflects or transmits must be planes on the scale of a few wavelengths. In most natural cases this is not perfectly true, and there is almost always roughness even at the wavelength scale. When there is a disagreement between the theory and reality, this is the most evident reason.

Table I. Summary of Computed Results

\begin{tabular}{|c|c|c|c|c|c|c|c|c|c|c|c|}
\hline$m$ & $\sigma$ & $\rho$ & $x$ & - & $Q$ & $a_{-1}$ & $a_{1}$ & $a_{2}$ & $a_{3}$ & $a_{4}$ & err \\
\hline 1.33 & 0.002 & 0.002 & & 1.0 & 1.001 & & & & & & \\
\hline 1.33 & 0.01 & 0.05 & & 1.0 & 1.003 & & & & & & \\
\hline 1.33 & 0.05 & 0.1 & & 1.0 & 1.01 & & & & & & \\
\hline 1.33 & 0.1 & 0.5 & & 1.0 & 1.13 & & & & & & \\
\hline 1.33 & 0.05 & 1.0 & & 1.0 & 1.14 & -0.897 & -16.84 & 20.11 & -10.64 & 2.065 & 5.4 \\
\hline 1.33 & 0.1 & 1.0 & & 1.0 & 1.24 & -0.882 & -16.46 & 19.83 & -10.48 & 2.027 & 4.4 \\
\hline 1.33 & 0.5 & 0.5 & & 1.0 & 1.37 & -1.741 & -31.77 & 37.69 & -20.08 & 3.907 & 16 \\
\hline 1.33 & 0.5 & 1.0 & & 1.0 & 1.73 & -1.116 & -19.53 & 23.17 & -12.20 & 2.355 & 5.3 \\
\hline 1.41 & 0.1 & 0.5 & & 1.0 & 1.13 & -1.307 & -25.47 & 30.77 & -16.82 & 3.362 & 18 \\
\hline 1.54 & 0.3 & 0.5 & & 1.0 & 1.26 & -0.087 & -4.738 & 7.927 & -5.582 & 1.359 & 6.2 \\
\hline 1.55 & 0.01 & 0.01 & & 1.0 & 1.002 & & & & & & \\
\hline 1.55 & 0.01 & 0.5 & & 1.0 & 1.03 & -1.908 & -28.44 & 29.58 & -14.77 & 2.843 & 13 \\
\hline 1.55 & 0.01 & 1:0 & & 1.0 & 1.04 & 0.112 & -2.104 & 3.944 & -2.785 & 0.681 & 1.7 \\
\hline 1.55 & 0.05 & 0.5 & & 1.0 & 1.09 & -1.160 & -19.94 & 22.14 & -11.73 & 2.370 & 10 \\
\hline 1.55 & 0.1 & 0.5 & & 1.0 & 1.13 & -0.580 & -12.80 & 15.84 & -9.154 & 1.965 & 8.7 \\
\hline 1.55 & 0.1 & 1.0 & & 1.0 & 1.24 & 0.166 & 0.176 & 1.448 & -1.482 & 0.423 & 1.4 \\
\hline 1.55 & 0.5 & 1.0 & & 1.0 & 1.73 & 0.135 & 0.571 & 1.019 & -1.308 & 0.407 & 1.6 \\
\hline $1.55+10^{-6} \mathrm{i}$ & 0.1 & 0.5 & 500 & 0.998 & 1.13 & -0.555 & -12.58 & 15.66 & -9.082 & 1.954 & 8.8 \\
\hline $1.55+10^{-3} \mathrm{i}$ & 0.1 & 0.5 & 500 & 0.98 & 1.13 & -0.560 & -12.57 & 15.65 & -9.083 & 1.956 & 8.8 \\
\hline $1.55+10^{-4} \mathrm{i}$ & 0.1 & 0.5 & 500 & 0.83 & 1.13 & -0.568 & -12.40 & 15.52 & -9.056 & 1.961 & 8.7 \\
\hline $1.55+10^{-3} \mathrm{i}$ & 0.1 & 0.5 & 500 & 0.23 & 1.13 & -0.824 & -13.71 & 15.74 & .8 .675 & 1.831 & 5.6 \\
\hline $1.55+0.01 i$ & 0.1 & 0.5 & 500 & 0.065 & 1.13 & -0.949 & -12.17 & 9.726 & -3.739 & 0.580 & 3.7 \\
\hline $1.55+0.1 \mathrm{i}$ & 0.1 & 0.5 & 500 & 0.067 & 1.13 & -0.929 & -12.05 & 9.615 & -3.690 & 0.570 & 3.4 \\
\hline $1.55+1.0 \mathrm{i}$ & 0.1 & 0.5 & 500 & 0.19 & 1.13 & -0.932 & -12.01 & 8.675 & -2.975 & 0.400 & 2.0 \\
\hline $1.55+10 \mathrm{i}$ & 0.1 & 0.5 & 500 & 0.94 & 1.13 & -0.897 & -12.01 & 8.134 & -2.580 & 0.312 & 1.3 \\
\hline $1.65+0.25 i$ & 0.1 & 1.7 & 31.2 & 0.054 & 1.31 & -0.0014 & -1.607 & 0.966 & -0.362 & 0.096 & 5.3 \\
\hline 1.70 & 0.1 & 0.5 & & 1.0 & 1.13 & -0.418 & -7.924 & 8.778 & -4.984 & 1.121 & 3.8 \\
\hline 2.0 & 0.1 & 0.5 & & 1.0 & 1.13 & -0.463 & -5.933 & 4.544 & -1.926 & 0.410 & 1.9 \\
\hline
\end{tabular}

Refractive index $m$, roughness parameters $\sigma, \rho$, size parameter $x=$ $k a$ ( $a=$ mean radius), single scattering albedo $\bar{\omega}_{0}, Q=\sigma_{\text {tot }} / \pi a^{2}\left(\sigma_{\text {tot }}=\right.$ total cross section). The next five columns give the parameter for the function fit [Eq. (28)] (not calculated for all particles), and the last one gives the rms error in percent. Note that these are only fitting errors and the true error can be larger. All these values are without diffraction.
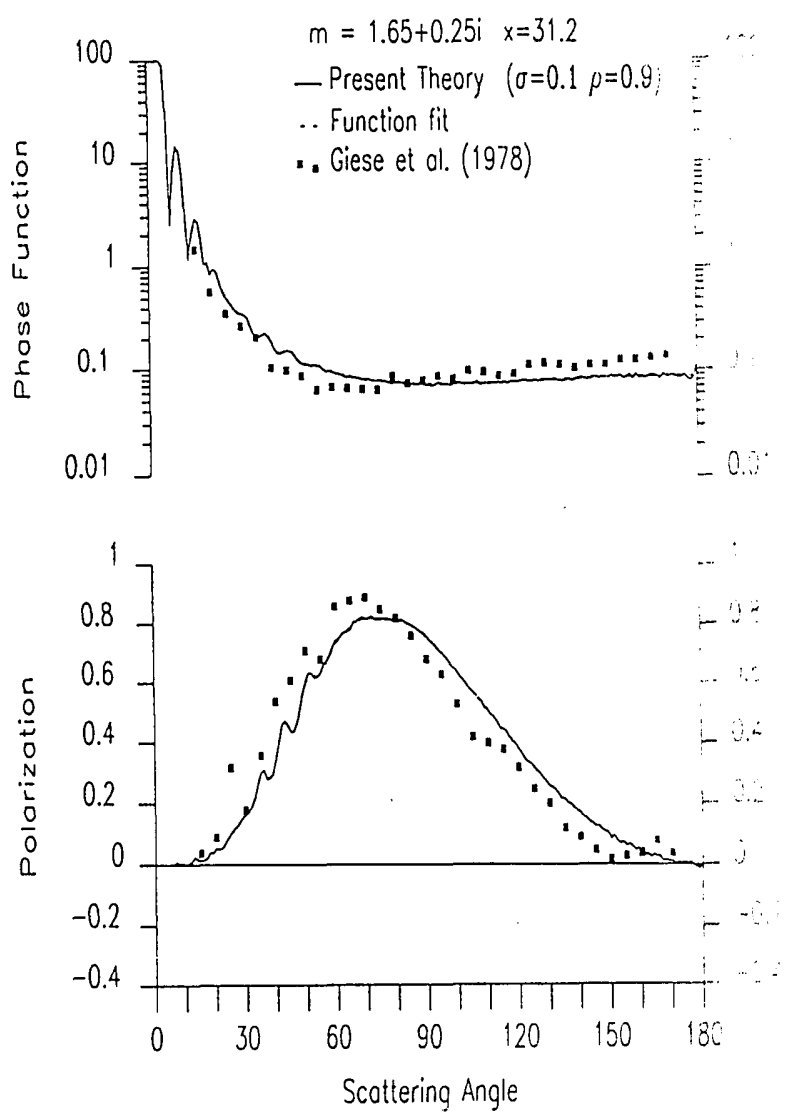

Fig. 6. Comparison of the present model with the experiment by Giese et al. ${ }^{14}$ Unlike in the previous figures, the diffraction is included in our results using a simple circular aperture theory. The dashed line in the upper figure (very near the solid line) is the function fit to our results [Eq. (28)].

Another small uncertainty comes from the use of a simple optical model of the medium. We assumed that the particle is internally homogeneous and isotropic and thus describable by a scalar refractive index. This may be an oversimplification, since many particles are to some degree anisotropic, birefringent, nonlinear, or consist of a mixture of different materials. The use of an average refractive index may or may not give correct results, depending on whether the inhomogeneities are large, small (Rayleigh criteria), or in between. ${ }^{16}$ Very often the surface of the particle is covered by a thin layer of different material (oxide, dust, or just more porous stuff).

The present model can be generalized to other types of stochastic particle having, for example, different statistics for the surface. One can guess, however, that the results are not very dependent on the detailed selection of particle model, if the particle is rough enough and the main features are kept similar. Thus a sufficiently perturbed spheroid, cube, or cylinder should give almost the same scattering as a similarly perturbed sphere. Only when the concavity becomes significantly high can we expect new features. When one wants to deal with even more irregular particles than those considered here, the next step is to construct a compact packing of several particles. One can 

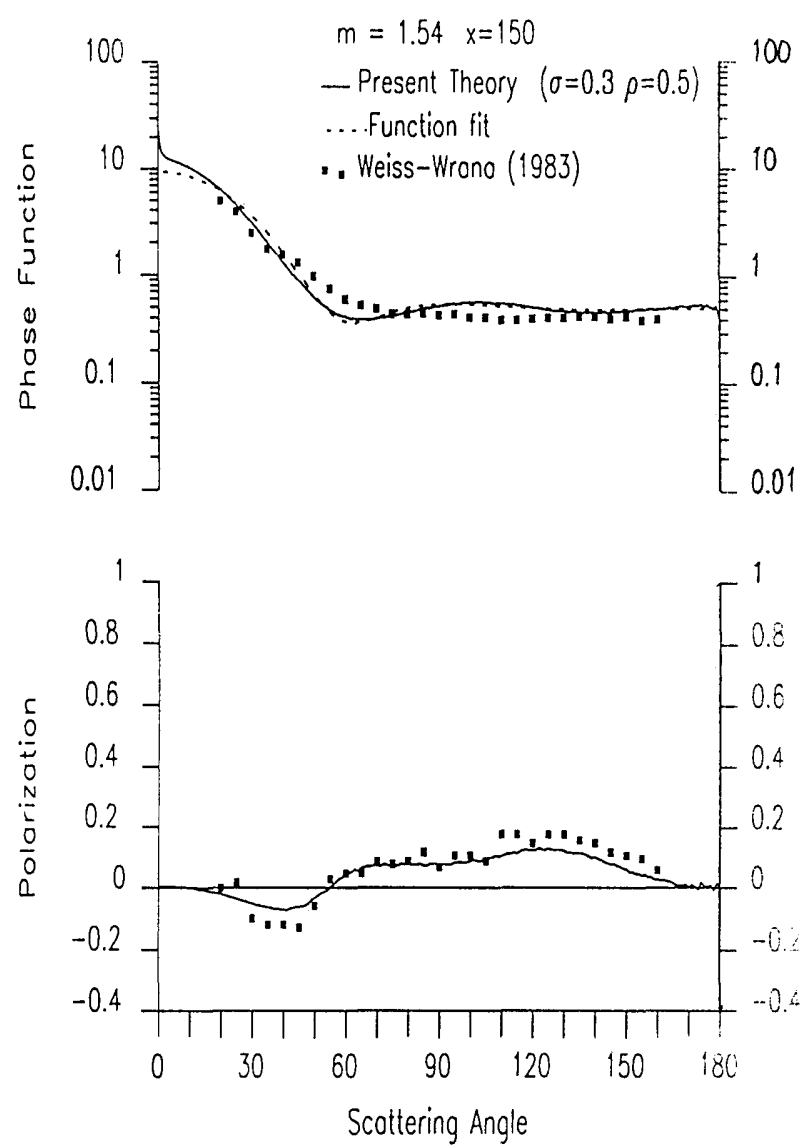

Fig. 7. Comparison of the present theory with experiment by Weiss-Wrana. ${ }^{15}$ The agreement is satisfactory.

also try to take the history of a ray better into account using multivariate statistics. It is, at least in principle, a rather straightforward task to extend the present model to include all these effects. The most serious problem is the rapidly increasing need for computing time.

Another modification to the present work would be to generalize the geometrical optical model with at least first order physical optics corrections. The way to do it might be to replace the Fresnel scattering of a surface element by some other plausible and more general law. Using perturbation theory the small scale roughness can be treated, and with the tangent plane (Kirchhoff) method perhaps intermediate irregularities could be included.

Some interesting applications of this theory to astronomy exist. With good reason one may expect a certain amount of planetary and interplanetary particles to be in the range of this theory. Giese et al. ${ }^{14}$ studied the properties of zodiacal light particles. Typically these particles are very irregular but the size is often in the difficult resonance region. In Saturn's rings the particles are large enough for geometrical optics to apply and consist mainly of pure water ice. In planetary regoliths the irregular particles play a major role. When formulating a radiative transfer theory for the surface of a regolith-covered body we must first know the phase function of the single particle, i.e., the subject of this paper. With our present model we, however, cannot explain either the opposition spike or the negative polarization in the backward direction that is observed for many airless bodies..$^{18}$ The assumption of fully irregular stochastic particles is not the whole truth, since there can be and usually are regular particles too. We need to add only a rather small amount of sharp-edged structure to a random surface to change the backscattering behavior significantly. Muinonen et al..$^{17}$ have calculated the scattering from various crystals and discuss more about the effects of such sharp-edged particles. Together with this paper, these calculations should significantly improve the ability to realistically model situations in planetary science and remote sensing such as those mentioned above.

William M. Irvine is partially supported by NASA grants NGL 22-010-023 and NAGW 1353.

\section{References}

1. C. F. Bohren and D. R. Huffman, Absorption and Scattering of Light by Small Particles (Wiley, New York, 1983).

2. H. C. van de Hulst, Light Scattering by Small Particles (Wiley, New York, 1957).

3. R. Schiffer, "The Effect of Surface Roughness on the Spectral Reflectance of Dielectric Particles. Application to Zodiacal Light," Astron. Astrophys. 148, 347-358 (1985).

4. F. G. Bass and I. M. Fuks, Wave Scattering from Statistically Rough Surfaces (Pergamon, New York, 1979).

5. R. Schiffer and K. O. Thielheim, "A Scattering Model for the Zodiacal Light Particles," Astron. Astrophys. 116, 1-9 (1982).

6. S. Mukai, T. Mukai, K. Weiss, and R. H. Zerull, "Scattering of Radiation by a Large Particle with a Random Rough Surface," Moon and Planets 26, 197-208 (1982).

7. D. W. Schuerman, Eds., Light Scattering by Irregularly Shaped Particles (Plenum, New York, 1980).

8. R. H. Giese and P. Lamy, Eds., Properties and Interactions of Interplanetary Dust (D. Reidel, Dordrecht, 1985).

9. K-N. Liou, An Introduction to Atmospheric Radiation (Academic, New York, 1980).

10. G. I. Marchuk, G. A. Mikhailov, M. A. Nazaraliev, R. A. Darbinjan, B. A. Kargin, and B. S. Elepov, The Monte-Carlo Methods in Atmospheric Optics (Springer-Verlag, New York, 1980).

11. L. L. House and L. W. Avery, "The Monte-Carlo Technique Applied to Radiative Transfer," J. Quant. Spectrosc. Radiat. Transfer 9, 1957-1991 (1967).

12. J. D. Jackson, Classical Electrodynamics (Wiley, New York, 1975), pp. 391-468.

13. B. G. Smith, "Geometrical Shadowing of a Random Rough Surface," IEEE Trans. Antennas Propag. AP-15, 668-671 (1967).

14. R. H. Giese, K. Weiss, R. H. Zerull, and T. Ono, "Large Fluffy Particles: A Possible Explanation of the Optical Properties of Interplanetary Dust," Astron. Astrophys. 65, 265-272 (1978).

15. K. Weiss-Wrana, "Optical Properties of Interplanetary Dust: Comparison with Light Scattering by Larger Meteoric and Terrestrial Grains," Astron. Astrophys. 126, 240-250 (1983).

16. F. T. Ulaby, R. K. Moore, and A. K. Fung, Microwave Remote Sensing (Addison-Wesley, Reading, MA, 1982), pp. 825-827.

17. K. Muinonen, K. Lumme, J. Peltoniemi, and W. M. Irvine, "Light Scattering by Randomly Oriented Crystals," Appl. Opt. 28, 3051-3060 (1989).

18. K. Lumme, K. Muinonen, J. Peltoniemi, H. Karttunen, and E. Bowell, "A Possible Explanation for the Anomalously Sharp Opposition Effects," Bull. Am. Astron. Soc. 19, 850 (1987).

19. M. Born and E. Wolf, Principles of Optics (Pergamon, New York, 1964). 